\title{
Cultural Competence, Safety, Humility, and Dexterity in Surgery
}

\author{
Charlotte B. Smith ${ }^{1}$ Laura N. Purcell ${ }^{2} \cdot$ Anthony Charles $^{2,3}$ (1)
}

Accepted: 29 November 2021 / Published online: 13 January 2022

(C) The Author(s), under exclusive licence to Springer Science+Business Media, LLC, part of Springer Nature 2022

\begin{abstract}
Purpose of Review As the United States' population diversifies, urgent action is required to identify, dismantle, and eradicate persistent health disparities. The surgical community must recognize how patients' values, beliefs, and behaviors are influenced by race, ethnicity, nationality, language, gender, socioeconomic status, physical and mental ability, sexual orientation, and occupation.

Recent Findings Lately, health disparities have been highlighted during the COVID-19 pandemic. Surgery is no exception, with notable disparities occurring in pediatric, vascular, trauma, and cardiac surgery. In response, numerous curricula and training programs are being designed to increase cultural competence and safety among surgeons.

Summary Cultural competence, safety, humility, and dexterity are required to improve healthcare experiences and outcomes for minorities. Various opportunities exist to enhance cultural competency and can be implemented at the medical student, resident, attending, management, and leadership levels.
\end{abstract}

This article is part of the Topical collection on Diversity in the Surgical Profession.

Anthony Charles

anthchar@med.unc.edu

1 University of North Carolina at Chapel Hill School of Medicine, Chapel Hill, CB, USA

2 Department of Surgery, University of North Carolina at Chapel Hill, Chapel Hill, CB, USA

3 Department of Surgery, University of north Carolina at Chapel Hill, 4008 Burnett Womack Building, Chapel Hill, CB 7228, USA
Keywords Healthcare disparities · Surgery · Cultural competence - Cultural humility · Cultural dexterity . Diversity

In the United States, by 2050 , approximately $50 \%$ of the population will belong to a minority racial/ethnic group [1]. The United States' increasing diversity brings opportunities and challenges for health care providers and policymakers as racial and ethnic disparities persist in today's healthcare system and have been highlighted during the COVID-19 pandemic. To deliver competent services, the health care community must recognize how individual values, beliefs, and behaviors are influenced by race, ethnicity, nationality, language, gender, socioeconomic status, physical and mental ability, sexual orientation, and occupation.

Cultural competence is defined as the ability of individuals and institutions to effectively deliver health care services that meet patients' socio-cultural and linguistic needs. Providers who value cultural competence aim to collaborate effectively with individuals from different cultures, improve healthcare experiences and outcomes and, most importantly, reduce health inequities and disparities.

Numerous studies across all surgical specialties have demonstrated pervasive racial and ethnic surgical disparities and exhibit the need for culturally competent surgical care:

- Pediatric Surgery: Black and non-White neonates with congenital diaphragmatic hernias had a $54 \%$ and $52 \%$ increased risk of mortality, respectively, than White neonates when controlled for pertinent covariates [2]. 
- Vascular Surgery: A study by Hughes et al. showed Black patients comprise $29 \%$ of patients undergoing a major lower extremity amputation, but only $12 \%$ of those undergoing an open surgical procedure and $10 \%$ undergoing an endovascular procedure for limb salvage compared to White patients [3].

- Trauma Surgery: Numerous studies have shown increase mortality in Black, Asian, and Hispanic trauma patients when controlling for injury severity and other pertinent covariates [4-9]. Black trauma patients have been shown to have worse post-trauma functional outcomes, particularly in the traumatic brain injury population [10-13].

- Cardiac Surgery: Black children undergoing cardiac surgery had $30 \%$ higher odds of referral to highmortality hospitals, and non-White children had an adjusted 36\% higher odds of mortality than their White counterparts [14]. Black and Hispanic adult patients are less likely than White patients to receive invasive cardiac procedures such as angioplasty, bypass surgery, and thrombolytic therapy when controlling for patient characteristics and socioeconomic status [15].

- Perioperative Management: Several studies have demonstrated significant racial disparities in postoperative pain management, particularly in the general surgical and orthopedic literature [16, 17].

Between 2014 and 2018, White surgeons constituted $77 \%$ of the surgical workforce, while Black and Hispanic surgeons made up $2 \%$ and $3 \%$, respectfully [18]. Accordingly, many racial inequities are associated with a failure of cultural understanding and implicit bias in cross-cultural situations. Yet, amongst surgeons who acknowledged such inequities, a majority (91.4\%) believed surgical disparities were solely attributable to patient factors instead of provider factors, social determinants of health, and power structures within healthcare [19].

This article examines the meaning of cultural competency and its utility as a critical element in addressing healthcare inequities. Finally, we explore the unique role surgeons can play in providing a culturally competent environment for all patients and surgical teams.

\section{Cultural Competence and Nomenclature}

Since the move to provide culturally competent care gained momentum during the American Civil Rights Movement in the 1960s, its terminology has seen numerous permutations [20]. It is essential to review these differences in terminology to lay the groundwork for an appropriate discussion.

\section{Cultural Competence}

In 1989, Cross et al. outlined cultural competence as "a set of congruent behaviors, attitudes, and policies that come together in a system, agency, or among professionals and enable that system, agency, or those professions to work effectively in cross-cultural situations." [21] This definition is beneficial, as it puts the responsibility on both individuals and healthcare systems to shift behaviors, attitudes, and policies to improve cross-cultural interactions. Finally, cultural competency is relevant to reduce patient disparities but also to improve health care team dynamics.

A narrow focus on cultural competency alone, however, has been criticized as it risks promoting a static understanding that centers on 'otherness,' perpetuates stereotypes, and ignores power differentials [22]. The unintended consequence of a limited focus on cultural competence positions the responsibility for poor health solely with the affected individuals or communities. As a result, this focus diminishes the impact of health professionals, the health care system, and broader socioeconomic structures on surgical health disparities seen today. Cultural competence is therefore considered essential but not adequate without cultural safety.

\section{Cultural Safety}

Cultural safety is concerned with deconstructing traditional power dynamics between the medical profession, patients and communities, to reduce bias and improve equity within the workforce and health care environments. Cultural safety asserts that health care and socioeconomic systems have created and perpetuated health inequities, and therefore attempts to address inequities by examining, critiquing, and dismantling those structures [22-24]. Cultural safety also affirms that it is not lack of cultural awareness driving health inequities but the direct result of unequal cultural power dynamics, unfair distribution of social determinants of health, marginalization, unexamined privilege, and institutional racism [25]. Thus, cultural safety creates a shift in focus, requiring the surgeon and healthcare system to learn about their beliefs and role in contributing to systemic health inequities, rather than focusing on the individual patient.

\section{Cultural Humility}

Cultural humility, a term coined in 1998, defines a dynamic lifelong introspection aiming to address power imbalance and enhance interpersonal sensitivity [26]. The term may be viewed as a muted rendering of cultural safety, adopting cultural competence and cultural safety benefits while 
choosing the more discreet word, humility. The choice of terminology also helps to create an appropriate mindset for HCPs engaging in cross-cultural situations. Inevitably, errors and missteps will occur in any attempt at building a productive and safe environment for HCPs and patients from minority groups. Humility leaves enough flexibility for surgeons to grow from mistakes and develop an ongoing commitment to learning about different cultures, intersectionality, and their own implicit biases. As such, many have embraced cultural humility as a supplement or replacement term for cultural competence [27].

\section{Cultural Dexterity}

Like humility, the semantics of dexterity provides many benefits. Surgeons use manual dexterity to apply their knowledge to patient care while navigating the unique circumstances of each surgical case [28]. Likewise, cultural dexterity allows surgical teams to apply socio-cultural awareness, emotional intelligence, and interpersonal skills in cross-cultural settings. It promotes adaptability over mastery, allowing surgeons to adjust to the unique needs of an individual patient [29]. While humility helps to create a flexible mindset, dexterity presents the idea of a personal toolbox that stores mental and physical skills related to providing culturally competent care.

\section{Cultural Awareness}

The preceding definitions depend on the surgical teams' ability to recognize the culture and interpret cross-cultural interactions. The National Center for Cultural Competence defines cultural awareness as being observant and conscious of similarities and differences among and between cultural groups [30]. Cultural awareness often requires individuals to become culturally self-aware: a critical understanding of how one's culture shapes their personality, beliefs, and biases. In becoming more cognizant about culture's influence on the self, surgeons must be prepared to be more sensitive and alert to other cultures. Without cultural awareness, the application of cultural competence, safety, humility, and dexterity become virtually meaningless Table 1.

Despite its drawbacks, cultural competence remains the dominant terminology used in current publications, medical education, and clinical settings [31-34]. However, the authors note that cultural competence is often used interchangeably and appropriates meaning from terms such as cultural humility, cultural awareness, and cultural dexterity.
Cultural Competence and the Delivery of Surgical Care

Cultural safety and competency are necessary components in addressing unacceptable health inequities, which disproportionately impact minority groups. Quality surgical care for patients is not limited to performing excellent surgery but requires making sound and culturally competent clinical judgments in a safe and inclusive clinical environment. Data shows that some parts of the community receive lesser-quality care according to the surgeon's standards and have lower health outcomes [4, 6, 35, 36]. The credibility of medical institutions rely on the public's trust [22, 37]. As leaders in the clinical and hospital settings, surgeons are uniquely positioned to advocate for surgical teams and healthcare system policies to eliminating health inequity. Surgeons can set the tone for delivering culturally competent operative and perioperative patient care.

Additionally, there is a clear need for culturally competent leadership in surgery. While more recently, medical schools and training programs are recruiting more women, racial and ethnic minorities, the slow surgical pipeline (with training spanning over a decade for many surgical specialties) does not lend itself to adjusting quickly [38-40]. These factors are compounded by slower career progression seen from medical school into residency and through associate professor to full professor, which show rapidly declining proportions of women and minorities in each advancing level. While many inspirational and aspiring young surgeons actively address surgical disparities and surgical cultural competence, the surgical community cannot just depend on trainees and young surgeons to provide this leadership. Effecting meaningful change needs to come from senior surgical leaders to change the culture of surgical residencies and departments to help minority surgeons thrive and deliver better surgical care to diverse patient groups.

\section{Phased Approach to Cultural Competence Training}

A critical step is linking the objective of cultural competence and safety to achieving health equity. This first step requires understanding that health inequities for Black, Indigenous, and People of Color (BIPOC) are unacceptable outcomes. Second, cultural competence must be a continuous reflective process, requiring ongoing selfassessment. Third, cultural competence requires a constant reassessment of power structures and privilege, taking meaning from cultural safety. Therefore, cultural 
Table 1 Cultural competence and nomenclature

\begin{tabular}{|c|c|c|}
\hline \multirow[t]{2}{*}{ Nomenclature } & \multicolumn{2}{|l|}{ Definition } \\
\hline & Benefits & Limitations \\
\hline $\begin{array}{l}\text { Cultural } \\
\text { competence }\end{array}$ & $\begin{array}{l}\text { "A set of congruent behaviors, attitudes, and policies that come to } \\
\text { that system, agency, or those professions to work effectively i } \\
\text { - Places responsibility on individuals and healthcare systems; } \\
\text { - Focuses on patient interactions and health care team dynamics }\end{array}$ & $\begin{array}{l}\text { gether in a system, agency, or among professionals and enable } \\
\text { n cross-cultural situations." [21] } \\
\text { - Risks promoting a static understanding that centers on } \\
\text { 'otherness,' perpetuates stereotypes, ignores power } \\
\text { differentials. [22] }\end{array}$ \\
\hline $\begin{array}{r}\text { Cultural } \\
\text { safety }\end{array}$ & $\begin{array}{l}\text { Asserts that health care and socioeconomic systems have createc } \\
\text { address inequities by examining, critiquing, and dismantling tl } \\
\text { - Pays attention to cultural power dynamics, social determinants } \\
\text { of health, marginalization, privilege, and institutional racism; } \\
\text { - Requires surgeons and healthcare systems to examine their role } \\
\text { in contributing to systemic health inequities }\end{array}$ & $\begin{array}{l}\text { and perpetuated health inequities, and therefore attempts to } \\
\text { ose structures. [22-24] }\end{array}$ \\
\hline $\begin{array}{l}\text { Cultural } \\
\text { humility }\end{array}$ & $\begin{array}{l}\text { Defines a dynamic lifelong introspection aimed at addressing po } \\
\text { - Helps to create an appropriate mindset for HCPs engaging in } \\
\text { cross-cultural situations; } \\
\text { - Embraced as a supplement or replacement term for cultural } \\
\text { competence. [27] }\end{array}$ & wer imbalance and enhance interpersonal sensitivity. [26] \\
\hline $\begin{array}{l}\text { Cultural } \\
\text { dexterity }\end{array}$ & $\begin{array}{l}\text { Allows surgical teams to adapt and apply sociocultural awarenes } \\
\text { cultural settings } \\
\text { - Promotes adaptability over mastery; } \\
\text { - Develops the framework of a personal toolbox that stores } \\
\text { mental and physical skills related to providing culturally } \\
\text { competent care }\end{array}$ & s, emotional intelligence, and interpersonal skills in cross- \\
\hline $\begin{array}{l}\text { Cultural } \\
\text { awareness }\end{array}$ & $\begin{array}{l}\text { Observes similarities and differences among and between cultura } \\
\text { interactions. [30] } \\
\text { - Requires individuals to become culturally self-aware } \\
\text { - Process of being more sensitive and alert to other cultures }\end{array}$ & groups by recognizing culture and interpreting cross-cultural \\
\hline
\end{tabular}

Summary of relevant definitions, benefits, and limitations of terms used interchangeably or in lieu of cultural competence

competence should not be limited to formal training curricula but also aligned across all training/practice environments, systems, structures, and policies [22].

\section{The Individual}

In its most basic form, culturally competent care is rooted in patient/provider interactions. The individual surgeon and surgical team members can still modify their behavior, evaluate their biases, and enhance the quality of their interactions with patients. Several guidelines include:

- Embracing a lifelong approach for gaining cultural competency. Just as culture continues to evolve, cultural competency does not have a discrete endpoint. Individuals can continually improve their cultural dexterity and awareness to meet the needs of their patients better.

- Adopting an open mentality fosters growth, curiosity, and humility when learning about one's own culture and others. For example, surgical providers can use the
ASSESS acronym to engage an appropriate mindset in cross-cultural interactions: Ask questions in a humble, safe manner; Seek self-awareness; Suspend judgment; Express kindness and compassion; Support a safe and welcoming environment, and Start where the patient is. [41]

- Magnifying an individual's impact through advocacy. Reaching out to family, friends, colleagues, and institutions can exponentially affect the number of individuals who seek to learn about cultural competency and integrate those practices in daily life.

\section{The Surgical Team (and Team Dynamics)}

We can similarly apply individual guidelines on cultural competence to surgical teams. An attitude shift gains more support if they become the behavioral norm within a group, and advocacy is more impactful when supported by a larger group.[42] As the recruitment, retention, and promotion of Black, Indigenous, and People of Color (BIPOC) in surgery 
Table 2 Phased approach to cultural competence training

\begin{tabular}{ll}
\hline Level & Cultural competence \\
\hline Individual & - Embrace a lifelong approach for gaining cultural competency; \\
& - Use the ASSESS acronym to engage an appropriate mindset in cross-cultural interactions; [41] \\
& - Advocate to engage family, friends, colleagues, and institutions \\
& - Promote team-based communication that fosters mutual respect, transparency, and productive feedback; \\
& - Set expectations for culturally aware teamwork and patient care; \\
Surgical team & - Partner with others to promote culturally safe and aware care from all aspects of the patient experience \\
& - Introduce the Provider Awareness and Cultural dexterity Toolkit for Surgeons (PACTS; [43] \\
& - Update policies relevant to cultural humility, reporting, and increasing representation in the hiring process; \\
Academic centers/ & - Create opportunities for providers to live and work outside their home culture and to facilitate cultural self- \\
institutions & awareness \\
& - Recruit diverse and multicultural learners; \\
Physician training & - Emphasize self-reflective learning, power imbalances, and the impact of provider behavior on health inequities in \\
programs & program curriculum
\end{tabular}

Summary of strategies to promote culturally competent care and health equity targeted at different levels in the healthcare system

improve, ensuring a culturally safe work environment is imperative. As such, certain cultural practices to encourage in teams are:

- Open communication. Good team-based communication fosters mutual respect, transparency, and productive feedback. Communication helps team members recognize implicit biases and institutionalize privilege, benefiting from a trusted outside perspective. Additionally, colleagues can be more receptive to feedback and avoid repeating mistakes in cross-cultural interactions.

- Expectations/Standards. Progress can be more effective when individuals work as a team. By setting expectations, a team can create a sense of shared responsibility, maintain greater focus, and leave less room for misunderstandings. Explicit expectations can also help standardize patient care across providers.

- Inter-professional approach. As previously touched upon, surgeons occupy a privileged leadership position within the surgical team. A partnership with other HCPs such as nurses, physician associates, and physical therapists can promote culturally safe and aware care from all aspects of the patient experience.

\section{Academic Centers/Institutions}

Any institution that provides patient care, from larger academic centers to smaller private practices, can initiate effective, culturally competent policies and behaviors. Institutional leadership can set the tone on issues such as health inequities and diversity and inclusion. They can also create and enforce standards, implement training requirements, and provide resources for their staff. Possible methods for action include:

- Training. The Provider Awareness and Cultural Dexterity Toolkit for Surgeons (PACTS) could be introduced to standardize cultural competency training for all current staff. Lee et al. noted that institutional support, participant engagement, and clear applicability to surgical practice are essential for this curricula to be successful. [43]

- Policy changes. Potential changes include updating the code of conduct to include cultural humility, promoting an open-door policy to facilitate reporting, applying the rules swiftly and equally to all staff members, and increasing representation in the hiring process.

- Surgical Volunteerism: Opportunities to live and work outside your home culture facilitate cultural selfawareness-a necessary first step for achieving cultural humility and dexterity.

\section{Physician Training Programs: Medical School, Residency and Fellowship}

Physician training programs must prioritize values to help create the next generation of culturally dexterous providers. This is the most sustainable way to ensure progress and eliminate current health disparities. Key areas of focus should include:

- Recruiting diverse and multicultural students. The surgical workforce is missing appropriate representation of minorities. While pipeline programs and recruitment efforts have made headway, much progress is still required to overcome the current deficit and 
build programs that facilitate retention, growth, and leadership opportunities [38, 44].

- Curriculum Changes. The American Association of Medical Colleges (AAMC) made cultural competence a core competency for pre-medical students. The Accreditation Council for Graduate Medical Education (ACGME) also made cross-cultural education a general competency for medical students and residents $[45,46]$. Teachings should emphasize its ongoing, self-reflective nature and recognize how power imbalances and provider behavior impact health inequities Table 2 .

\section{Conclusion}

Surgeons must continue to care about cultural competence with each patient encounter, and mastering measures to improve cultural competence will help alleviate healthcare disparities and improve health care outcomes in surgical patient populations. Efforts must begin early in the pipeline to attract qualified racial, ethnic, and gender minorities to the field; actively improve the surgical culture to retain and promote highly motivated and skilled competent minorities in surgery; and advocate for culturally competent healthcare policy to improve the access and outcomes for surgical care nationwide. Cultural competence must be required for all surgeons and surgical trainees, regardless of race, ethnicity, nationality, language, gender, socioeconomic status, physical and mental ability, sexual orientation. Various opportunities exist to enhance cultural competency at the medical student, resident, attending, management, and leadership levels.

Author Contributions All authors contributed to the conception and writing of this review.

Funding The authors have no funding to acknowledge in regards to the preparation of this manuscript.

\section{Data Availability (N/A).}

Code Availability (N/A).

\section{Compliance with Ethical Guidelines}

Conflict of interest The authors have no proprietary or financial interest in reporting any resources or concepts discussed in this review.

\section{Ethical Approval (N/A).}

\section{Consent to Participate (N/A).}

Consent for Publication All authors approve of the final version of this manuscript and its publication in this journal.

\section{References}

Papers of particular interest, published recently, have been highlighted as:

- Of importance

-• Of major importance

1. Colby SL, Ortmann JM. Projections of the size and composition of the U.S. population: 2014 to 2060. Suitland: US Census Bureau; 2015.

2. Sola JE, Bronson SN, Cheung MC, et al. Survival disparities in newborns with congenital diaphragmatic hernia: a national perspective. J Pediatr Surg. 2010;45:1336-42. https://doi.org/10. 1016/j.jpedsurg.2010.02.105.

3. Hughes K, Boyd C, Oyetunji T, et al. Racial/ethnic disparities in revascularization for limb salvage: an analysis of the National Surgical Quality Improvement Program database. Vasc Endovascular Surg. 2014;48:402-5. https://doi.org/10.1177/ 1538574414543276.

4. Bowman SM, Martin DP, Sharar SR, Zimmerman FJ. Racial disparities in outcomes of persons with moderate to severe traumatic brain injury. Med Care. 2007;45:686-90. https://doi. org/10.1097/MLR.0b013e31803dcdf3.

5. Maybury RS, Bolorunduro OB, Villegas C, et al. Pedestrians struck by motor vehicles further worsen race- and insurancebased disparities in trauma outcomes: the case for inner-city pedestrian injury prevention programs. Surgery. 2010;148:202-8. https://doi.org/10.1016/j.surg.2010.05.010.

6. Hakmeh W, Barker J, Szpunar SM, et al. Effect of race and insurance on outcome of pediatric trauma. Acad Emerg Med. 2010;17:809-12. https://doi.org/10.1111/j.1553-2712.2010. 00819.x.

7. Rosen H, Saleh F, Lipsitz S, et al. Downwardly mobile: the accidental cost of being uninsured. Arch Surg. 2009;144:1006-11. https://doi.org/10.1001/archsurg.2009.195.

8. Oyetunji TA, Crompton JG, Ehanire ID, et al. Multiple imputation in trauma disparity research. J Surg Res. 2011;165:e37-41. https://doi.org/10.1016/j.jss.2010.09.025.

9. Falcone RA, Brown RL, Garcia VF. Disparities in child abuse mortality are not explained by injury severity. J Pediatr Surg. 2007;42:1031-6. https://doi.org/10.1016/j.jpedsurg.2007.01.038.

10. Arango-Lasprilla JC, Rosenthal M, Deluca J, et al. Functional outcomes from inpatient rehabilitation after traumatic brain injury: how do Hispanics fare? Arch Phys Med Rehabil. 2007;88:11-8. https://doi.org/10.1016/j.apmr.2006.10.029.

11. Arango-Lasprilla JC, Rosenthal M, Deluca J, et al. Traumatic brain injury and functional outcomes: does minority status matter? Brain Inj. 2007;21:701-8. https://doi.org/10.1080/ 02699050701481597.

12. Staudenmayer KL, Diaz-Arrastia R, de Oliveira A, et al. Ethnic disparities in long-term functional outcomes after traumatic brain injury. J Trauma. 2007;63:1364-9. https://doi.org/10.1097/TA. 0b013e31815b897b.

13. Haider AH, Efron DT, Haut ER, et al. Black children experience worse clinical and functional outcomes after traumatic brain injury: an analysis of the National Pediatric Trauma Registry. J Trauma. 2007;62:1259-62. https://doi.org/10.1097/TA. 0b013e31803c760e.

14. Chan T, Pinto NM, Bratton SL. Racial and insurance disparities in-hospital mortality for children undergoing congenital heart surgery. Pediatr Cardiol. 2012;33:1026-39. https://doi.org/10. 1007/s00246-012-0221-z.

15. Ford ES, Cooper RS. Implications of race/ethnicity for health and health care use: racial/ethnic differences in healthcare utilization 
of cardiovascular procedures: a review of the evidence. Health Serv Res. 1995;30(1):237-52.

16. Mossey JM. Defining racial and ethnic disparities in pain management. Clin Orthop Relat Res. 2011;469:1859-70. https://doi. org/10.1007/s11999-011-1770-9.

17. Lawrence AE, Deans KJ, Chisolm DJ, et al. Racial disparities in receipt of postoperative opioids after pediatric cholecystectomy. J Surg Res. 2020;245:309-14. https://doi.org/10.1016/j.jss.2019. 07.043 .

18. Noel OF, Berg A, Onyango N, Mackay DR. Ethnic and gender diversity comparison between surgical patients and caring surgeons. Plast Reconstr Surg Glob Open. 2020;8:e3198. https://doi. org/10.1097/GOX.0000000000003198.

19. Britton BV, Nagarajan N, Zogg CK, et al. Awareness of racial/ ethnic disparities in surgical outcomes and care: factors affecting acknowledgment and action. Am J Surg. 2016;212:102-108.e2. https://doi.org/10.1016/j.amjsurg.2015.07.022.

20. - Chin JL. Culturally competent health care. Public Health Rep. 2000;115:25-33. This goes into a detailed discussion of the orgins of the nomenclature for cultural competence.

21. Cross TL, Bazron BJ, Dennis KWD, Isaacs MR. Towards a culturally competent system of care: a monograph on effective services for minority children who are severely emotionally disturbed. Washington, DC: CASSP Technical Assistance Center; 1989.

22. Curtis E, Jones R, Tipene-Leach D, et al. Why cultural safety rather than cultural competency is required to achieve health equity: a literature review and recommended definition. Int $\mathbf{J}$ Equity Health. 2019;18:174. https://doi.org/10.1186/s12939-0191082-3.

23. Papps E, Ramsden I. Cultural safety in nursing: the New Zealand experience. Int J Qual Health Care. 1996;8:491-7. https://doi.org/ 10.1093/intqhe/8.5.491.

24. Laverty M, McDermott DR, Calma T. Embedding cultural safety in Australia's main health care standards. Med J Aust. 2017;207:15-6. https://doi.org/10.5694/mja17.00328.

25. Robson B, Harris R (2007) Hauora: Māori Standards of Health IV. A Study of the Years 2000-2005. Wellington: Te Rōpu Rangahau Hauora a Eru Pōmare.

26. Tervalon M, Murray-García J. Cultural humility versus cultural competence: a critical distinction in defining physician training outcomes in multicultural education. J Health Care Poor Underserved. 1998;9:117-25. https://doi.org/10.1353/hpu.2010.0233.

27. Campinha-Bacote J (2019) Cultural competemility: a paradigm shift in the cultural competence versus cultural humility debatePart I. OJIN 24.

28. The hands have it: the importance of manual dexterity in medicine-International Museum of surgical science. Available at https://imss.org/2020/12/14/the-hands-have-it-the-importance-ofmanual-dexterity-in-medicine/. Accessed on 3 Aug 2021

29. Hazard P. Cultural dexterity: the global talent solution. Los Angeles: Korn Ferry Institute; 2014.

30. Gilbert J, Goode TD, Dunne C (2007) CURRICULA ENHANCEMENT MODULE: National Center for Cultural Competence. Georgetown University Child Development Center University, Center for Excellence in Developmental Disabilities.

31. Darivemula S, Bhatia R, Bhumi S. Dancing doctors: exploring the potential of cultural dance education to improve cultural competency and clinical communication. Public Health. 2021;195:22-3. https://doi.org/10.1016/j.puhe.2021.03.019.
32. Slobodin O, Kula Y, Clempert N, Cohen O. Building capacity in times of crisis: increasing cultural competence of healthcare professionals in the context of the COVID-19. J Clin Nurs. 2021. https://doi.org/10.1111/jocn.15897.

33. Shepherd SM, Willis-Esqueda C, Newton D, et al. The challenge of cultural competence in the workplace: perspectives of healthcare providers. BMC Health Serv Res. 2019;19:135. https:// doi.org/10.1186/s12913-019-3959-7.

34. Pettersson S, Holstein J, Jirwe M, et al. Cultural competence in healthcare professionals, specialized in diabetes, working in primary healthcare-a descriptive study. Health Soc Care Commun. 2021. https://doi.org/10.1111/hsc. 13442 .

35. •• Changoor NR, Udyavar NR, Morris MA, et al. Surgeons' perceptions toward providing care for diverse patients: the need for cultural dexterity training. Ann Surg. 2019;269:275-82. https://doi.org/10.1097/SLA.0000000000002560. Explores cultrual competency and dexterity as it relates to surgeons.

36. Erkmen CP, Ortmeyer KA, Pelletier GJ, et al. An approach to diversity and inclusion in cardiothoracic surgery. Ann Thorac Surg. 2021;111:747-52. https://doi.org/10.1016/j.athoracsur. 2020.10.056.

37. Ronald MM, Aramoana JKA, Hill AG. Surgeons and cultural safety and cultural competency: the road to transformation. ANZ J Surg. 2020. https://doi.org/10.1111/ans.16379.

38. Bingmer K, Ofshteyn A, Bliggenstorfer JT, et al. Where is the leak in the surgeon pipeline? Am J Surg. 2020;220:1174-8. https://doi.org/10.1016/j.amjsurg.2020.06.048.

39. Abelson JS, Chartrand G, Moo T-A, et al. The climb to break the glass ceiling in surgery: trends in women progressing from medical school to surgical training and academic leadership from 1994 to 2015. Am J Surg. 2016;212:566-572.e1. https://doi.org/ 10.1016/j.amjsurg.2016.06.012.

40. Dorsey C, Ross E, Appah-Sampong A, et al. Update on workforce diversity in vascular surgery. J Vasc Surg. 2020. https://doi. org/10.1016/j.jvs.2020.12.063.

41. Boesen L (2012) Creating connections through cultural humility. Available at http://www.lisaboesen.com/creating-connectionsthrough-cultural-humility/. Accessed on 4 Aug 2021.

42. Chung A, Rimal RN. Social norms: a review. RCR. 2016;4:1-28. https://doi.org/10.12840/issn.2255-4165.2016.04.01.008.

43. Lee GJ, Ortega G, Reidy E, et al. A qualitative analysis of surgical faculty and surgical resident perceptions of potential barriers to implementing a novel surgical education curriculum. J Surg Educ. 2021;78:896-904. https://doi.org/10.1016/j.jsurg. 2020.09.023.

44. Osseo-Asare A, Balasuriya L, Huot SJ, et al. Minority resident physicians' views on the role of race/ethnicity in their training experiences in the workplace. JAMA Netw Open. 2018;1:e182723. https://doi.org/10.1001/jamanetworkopen.2018. 2723.

45. Shah SS, Sapigao FB, Chun MBJ. An overview of cultural competency curricula in ACGME-accredited general surgery residency programs. J Surg Educ. 2017;74:16-22. https://doi.org/ 10.1016/j.jsurg.2016.06.017.

46. The Anatomy of Cultural Competence. In: AAMC. Available at https://students-residents.aamc.org/anatomy-applicant/anatomycultural-competence. Accessed on 7 Aug 2021

Publisher's Note Springer Nature remains neutral with regard to jurisdictional claims in published maps and institutional affiliations. 